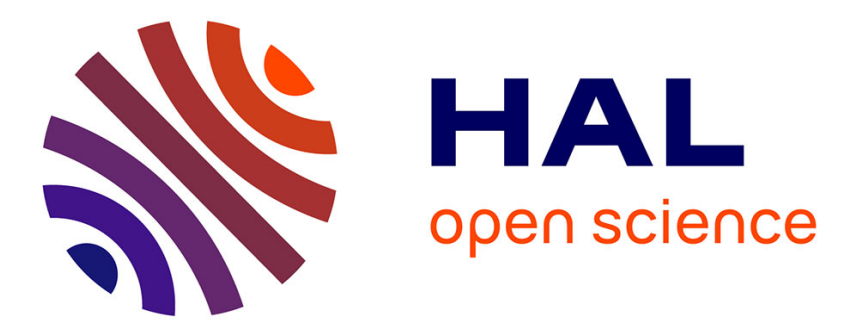

\title{
Relations entre les caractéristiques des laits de troupeaux, les pratiques d'élevage et les systèmes d'exploitation dans la zone de production du Beaufort
}

\author{
B. Dubeuf
}

\section{- To cite this version:}

B. Dubeuf. Relations entre les caractéristiques des laits de troupeaux, les pratiques d'élevage et les systèmes d'exploitation dans la zone de production du Beaufort. Productions Animales, 1995, 8 (2), pp.105-116. hal-00896108

\section{HAL Id: hal-00896108 \\ https://hal.science/hal-00896108}

Submitted on 1 Jan 1995

HAL is a multi-disciplinary open access archive for the deposit and dissemination of scientific research documents, whether they are published or not. The documents may come from teaching and research institutions in France or abroad, or from public or private research centers.
L'archive ouverte pluridisciplinaire HAL, est destinée au dépôt et à la diffusion de documents scientifiques de niveau recherche, publiés ou non, émanant des établissements d'enseignement et de recherche français ou étrangers, des laboratoires publics ou privés. 
INRA Prod. Anim., 1995, 8 (2), 105-116

\section{B. DUBEUF}

INRA Unité de Recherches sur les Systèmes Agraires et le Développement, Route de Saint-Cyr, 78026 Versailles Cedex
Relations entre les caractéristiques des laits de troupeaux, les pratiques d'élevage et les systèmes d'exploitation dans la zone de production du Beaufort

\begin{abstract}
L'Union des Producteurs de Beaufort et le Syndicat de défense gèrent la qualité du fromage Beaufort avec le souci de préserver ses qualités intrinsèques et de respecter son " authenticité » en cherchant à consolider la liaison terroir-races bovines-produit. La diversité des systèmes de production laitière est reconnue et son maintien est souhaitable sous réserve que ces systèmes reposent sur des techniques et des savoir-faire traditionnels respectant l'environnement.
\end{abstract}

Devant les risques de diminution du nombre de petits producteurs, de sous-exploitation de certains espaces et d'appauvrissement de la diversité des systèmes d'exploitation, l'Union des Producteurs de Beaufort (UPB) s'interroge sur une gestion de la qua-

\section{Résumé}

Une typologie des laits livrés par 129 des bassins de collecte de deux coopératives fromagères de la filière Beaufort a permis de mettre en évidence une diversité d'associations des caractéristiques des laits livrés (taux protéique, taux butyreux, cellules, flore totale, répartition annuelle de la livraison, volume annuel). A partir des données recueillies dans 34 exploitations laitières, des pratiques d'élevage ont été associées aux différents types de lait. L'étude confirme l'importance des facteurs alimentaires et de la période des vêlages dans l'évolution de la composition chimique du lait, l'importance des problèmes d'hygiène de la machine à traire dans la présence de germes totaux et la multiplicité des facteurs d'élevage associés à l'augmentation des leucocytes dans le lait. La diversité des associations des caractéristiques des laits sous-tend celle des pratiques associées et de la maîtrise par les producteurs de l'ensemble du système d'élevage. L'existence des liaisons entre les types de laits et les systèmes d'exploitation montre l'intérêt de proposer des actions de qualité adaptées à la diversité des exploitations laitières. lité du lait au sein de la filière qui puisse répondre aux attentes des coopératives fromagères et s'adapter à la diversité des exploitations laitières. Actuellement, les types de conseil pratiqués en matière de production laitière sont variés en raison de la diversité des agents pouvant intervenir sur ce domaine (contrôleurs laitiers, conseillers agricoles, techniciens de laiterie, commerciaux, vétérinaires...). Pour permettre l'aide à la décision d'actions adaptées à la diversité des problèmes de qualité du lait et des systèmes d'exploitation, les coopératives ont besoin de connaître leurs producteurs à travers leur système d'exploitation et le type de laits livrés, défini à partir de critères de qualité que recherchent les coopératives.

Certains critères, tels que la composition chimique du lait et ses teneurs en germes totaux et en leucocytes, ont fait l'objet de nombreux travaux expérimentaux ainsi que d'études dans des exploitations laitières. Leurs facteurs de variation sont bien connus. La composition chimique du lait de troupeau 
varie sous l'effet conjugué de nombreux facteurs physiologiques, saisonniers et alimentaires (Hoden et al 1985, Agabriel et al 1990 et 1993, Coulon et al 1991). Les germes totaux dans le lait ont trois origines: la flore de la mamelle, l'infection mammaire et l'environnement (Pankey 1989). Une teneur élevée en germes totaux est principalement associée à des problèmes d'hygiène du matériel de traite, à l'absence de nettoyage de la mamelle et à des problèmes de refroidissement du lait (Galton et al 1986, 1989). Un taux élevé de leucocytes témoigne de l'existence d'une mammite, dont l'apparition et le développement dépendent de nombreux facteurs environnementaux et individuels (Barnouin et al 1986, Pluvinage et al 1991). Mais peu de travaux de recherche ont étudié simultanément l'ensemble de ces caractéristiques du lait au niveau des exploitations. Or, pour les coopératives fromagères, le concept de qualité du lait comporte un ensemble de critères qui prennent en considération la structure de livraison du lait et ses caractéristiques intrinsèques ainsi que le système de production et le savoir-faire du producteur.

L'objectif de cette étude, réalisée dans deux coopératives fromagères de la filière du Beaufort, est de mettre en évidence la diversité des laits livrés définis par leur composition chimique, leur qualité sanitaire, leur volume annuel et l'évolution annuelle de ce volume et de la relier aux pratiques des livreurs. Ces pratiques ont été analysées dans le contexte général du fonctionnement d'exploitation, afin d'identifier les relations existant entre les types de laits et les systèmes d'exploitation. Il s'agit de fournir aux coopératives les éléments leur permettant de formuler un diagnostic sur les problèmes de qualité du lait dans leur bassin de collecte et de construire un questionnement pour un conseil adapté à la diversité des producteurs.

\section{1 / Démarche}

\section{1 / Typologie des laits livrés}

La typologie des laits a été réalisée à partir des données mensuelles collectées durant l'année 1991 dans les 129 exploitations adhérentes aux deux coopératives fromagères étudiées. Cette typologie a été élaborée à partir d'une analyse factorielle des correspondances (AFC) sur les critères suivants, découpés en classes (tableau 1):

- la structure de livraison du lait. Ce critère associe deux facteurs ayant une incidence économique pour les coopératives: les périodes de livraison à la coopérative durant l'année et le pourcentage de lait livré l'été. La structure de livraison a été obtenue en croisant deux critères: le nombre de mois de livraison au cours de l'année et le nombre de mois de livraison durant la période de juin à septembre. Les livreurs permanents ont été départagés selon la proportion de lait livré sur la période allant de juin à octobre (période d'alpage).

- le volume annuel de lait livré, indicateur du poids économique du producteur au sein de la coopérative.

- les moyennes pondérées annuelles du taux protéique et du taux butyreux. Les seuils des classes du volume annuel de lait livré et des moyennes annuelles pondérées des taux ont été établis dans l'objectif d'équilibrer les effectifs des classes.

- le taux de leucocytes et le taux de germes totaux. Les données mensuelles ont été codées selon les classes retenues dans la grille de paiement du lait. Dans un second temps, chaque livreur a été classé, pour le critère considéré, selon la fréquence observée des différents codes au cours de l'année. La classe 1 correspond à la norme de qualité recherchée en France et dans la C.E.E. Les autres classes ont été définies après observation de l'ensemble du fichier afin de respecter un compromis entre un équilibre des effectifs par classe et une délimitation des paliers montrant bien une intensification des problèmes d'hygiène.

Une classification hiérarchique par ascendance, réalisée sur les coordonnées des individus des 5 premiers axes de l'AFC, a permis de donner une première typologie des laits livrés. L'interprétation des différents types nous a amenés à vérifier l'appartenance de chaque individu au type de lait considéré. Ainsi des individus ont été reclassés manuellement à chaque fois que leurs caractéristiques s'apparentaient davantage à un autre type, ou que leur reclassement permettait de mieux préciser les caractéristiques des types.

\section{2 / Les pratiques associées aux types de laits livrés}

La typologie des laits livrés a permis de caractériser 7 types de laits, qui ont servi de références pour choisir un échantillon de producteurs dont les livraisons étaient représentatives de la diversité des laits livrés dans les deux coopératives. Le rapport entre les effectifs des coopératives a été, de plus, respecté. Parmi les 129 producteurs, 34 ont été enquêtés. Les informations recueillies ont porté sur la structure de l'exploitation (foncier, bâtiments, matériels de récolte, main d'œuvre, troupeau, type de produits commercialisés...), sur les pratiques relatives aux ressources fourragères (fertilisation, production de fourrages, achat de fourrages), et sur les pratiques d'élevage réparties en quatre catégories (Landais 1987) : les pratiques d'agrégation déterminant la composition des groupes d'animaux (pratiques de mise en pension et/ou prise en pension durant l'été et/ou l'hiver); les pratiques de renouvellement (élevage des génisses, achats de génisses prêtes, de génisses de 8 jours, de vaches laitières); les pratiques de conduite (alimentation estivale et hivernale, reproduction, santé) ; les pratiques d'exploitation (réforme, traite, vente de reproducteurs, fabrication fermière). 
Tableau 1. Classes des caractéristiques du lait.

\begin{tabular}{|c|c|c|}
\hline Caractéristique & Classe & \\
\hline \multirow[t]{6}{*}{ Structure de livraison } & LV1 & livraison l'hiver durant 7 mois au plus \\
\hline & $\mathrm{LV} 2$ & livraison durant 8 à 10 mois sauf l'été \\
\hline & LV3 & livraison toute l'année avec un pourcentage de lait d'été $<40 \%$ \\
\hline & LV4 & livraison toute l'année avec un pourcentage de lait d'été $>40 \%$ \\
\hline & LV5 & livraison durant 8 à 10 mois sauf quelques mois d'hiver \\
\hline & LV6 & livraison l'été \\
\hline \multirow{5}{*}{ Volume annuel livré } & LT1 & moins de $10000 \mathrm{~kg}$ de lait \\
\hline & LT2 & entre 10000 et $20000 \mathrm{~kg}$ de lait \\
\hline & LT3 & entre 20000 et $50000 \mathrm{~kg}$ de lait \\
\hline & LT4 & entre 50000 et $100000 \mathrm{~kg}$ de lait \\
\hline & LT5 & $100000 \mathrm{~kg}$ de lait et plus \\
\hline \multirow[t]{4}{*}{ TP annuel pondéré } & TP1 & inférieur à $30 \mathrm{~g} / \mathrm{kg}$ \\
\hline & TP2 & de 30 à $31 \mathrm{~g} / \mathrm{kg}$ \\
\hline & $\mathrm{TP} 3$ & de 31 à $32 \mathrm{~g} / \mathrm{kg}$ \\
\hline & TP4 & $32 \mathrm{~g} / \mathrm{kg}$ et plus \\
\hline \multirow{4}{*}{ TB annuel pondéré } & TB1 & moins de $34 \mathrm{~g} / \mathrm{kg}$ \\
\hline & TB2 & de 34 à $36 \mathrm{~g} / \mathrm{kg}$ \\
\hline & TB3 & de 36 à $38 \mathrm{~g} / \mathrm{kg}$ \\
\hline & TB4 & $38 \mathrm{~g} / \mathrm{kg}$ et plus \\
\hline \multirow[t]{3}{*}{ Germes totaux ${ }^{11}$} & BA1 & toutes les données mensuelles sont codées en 3 \\
\hline & $\begin{array}{l}\text { BA2 } \\
\text { BA3 }\end{array}$ & $\begin{array}{l}\text { une seule donnée codée en } 2 \text {, le reste des données codé en } 3 \text {. Sont concernés } \\
\text { par cette classe les laits ayant un minimum de } 7 \text { données mensuelles par an } \\
\text { des données codées en } 2 \text { et } 1 \text { (au maximum } 3 \text { données) }\end{array}$ \\
\hline & BA4 & un tiers des données au minimum sont codées en 1 et/ou 2 \\
\hline \multirow{5}{*}{ Leucocytes ${ }^{(2)}$} & LE1 & toutes les données mensuelles sont codées en 1 \\
\hline & LE2 & $\begin{array}{l}\text { données codées en } 2: \text { moins de } 25 \% \text { du nombre de données mensuelles } \\
\text { sur l'année } 1991 ; \text { données codées en } 1: 75 \% \text { et plus }\end{array}$ \\
\hline & LE3 & $\begin{array}{l}25 \%<\text { données } 2<75 \% \text {, le reste en données } 1 \text { ou des données } 1,2,3 \\
\text { (au maximum } 2 \text { données codées } 3 \text { ) (somme pondérée des données } \% \text { somme } \\
\text { des données }<1,33 \text { ) }\end{array}$ \\
\hline & LE4 & $\begin{array}{l}\text { données } 2>75 \% \text {, le reste en données } 1 \text { ou des données } 1,2,3 \text { (au maximum } \\
3 \text { données codées } 3)(1,33 \leqslant \text { somme pondérée des données/somme des données } \\
<1,75)\end{array}$ \\
\hline & LE5 & des données $1,2,3(1,75 \geqslant$ somme pondérée des données/somme des données) \\
\hline
\end{tabular}

(1) Code $3:$ moins de 100000 germes / ml, code $2:$ de 100000 à 500000 , code $1:$ plus de 500000.

(2) Code $1:$ moins de 400000 cellules $/ \mathrm{ml}$, code $2:$ de 400000 à 750000 , code $3:$ plus de 750000 .

${ }_{(*)}^{*}$ La somme pondérée des données $=\mathrm{x}+2 \mathrm{y}+3 \mathrm{z}(\mathrm{x}:$ nombre de données $1, \mathrm{y}:$ nombre de données $2, \mathrm{z}:$ nombre de données 3$)$.

Une AFC a été réalisée sur l'ensemble des pratiques, afin de mettre en évidence des associations. Seules les modalités dont la contribution était au moins égale à 1,5 fois la moyenne des contributions pour chacun des axes ont été prises en compte dans l'interprétation des axes. Pour identifier les pratiques associées aux types de lait, la variable «type de lait " a été introduite comme variable supplémentaire de l'AFC.

\section{3 / Relations entre types de laits et systèmes d'exploitation}

L'AFC a mis en évidence des liaisons entre pratiques. Dans les 34 exploitations enquêtées, l'analyse des pratiques liées entre elles et de leur cohérence avec d'autres facteurs de l'exploitation (structures, atouts et contraintes géographiques, techniques et économiques) a permis de dégager plusieurs systèmes d'exploitation. Linterprétation des relations entre types de laits et systèmes d'exploitation a été faite en croisant les deux typologies.

\section{2 / Résultats}

\section{1 / Les types de lait et les pratiques associées (tableau 2)}

Les 129 laits livrés se répartissent dans 7 types définis par des associations de critères de qualité (tableau 3 ).

Les laits des types 1 et $\mathbf{2}$ sont livrés de décembre à juin, en quantité faible mais relativement constante. Le type 1 est caractérisé par de très faibles TP et TB annuels et une évolution des TP et TB moyens mensuels comparable à celle d'une lactation dont le pic aurait lieu en janvier-février. Le type 2 présente des minima plus élevés d'un $\mathrm{g} / \mathrm{kg}$ pour le TP et de $3 \mathrm{~g} / \mathrm{kg}$ pour le TB et une variabilité importante des TP et TB moyens annuels entre livreurs. Ces deux types se différencient par leur teneur en leucocytes (faible pour le type 1, élevée pour le type 2) (figure 1). La teneur en germes totaux est satisfai- 
Tableau 2. Pratiques associées aux types de lait.

\begin{tabular}{|c|c|c|c|c|c|}
\hline Types de lait & & 1 et 2 & 3 et 4 & 5 et 6 & 7 \\
\hline Effectif des exploitations & $\mathrm{n}=34$ & 10 & 8 & 10 & 6 \\
\hline Mise à l'estive du troupeau & 11 & 8 & & 3 & \\
\hline Stabilité du troupeau laitier & 12 & & 8 & 2 & 2 \\
\hline Prise à l'estive des laitières & 5 & $1(\mathrm{f})$ & & 3 & 1 \\
\hline Mise à l'hiverne des laitières & 6 & $1(f)$ & & 2 & 3 \\
\hline Pas d'alpage & 13 & 8 & 4 & 1 & \\
\hline Pratique de l'inalpage & 21 & $2(\mathrm{f})$ & 4 & 9 & 6 \\
\hline Vêlage d'octobre février & 10 & 8 & 1 & & 1 \\
\hline Vêlage d'octobre à mars & 6 & & 4 & 2 & \\
\hline Vêlage d'octobre à juin & 13 & $2(\mathrm{f})$ & 3 & 7 & 2 \\
\hline Vêlage toute l'année & 5 & & & 1 & 4 \\
\hline Mauvaise maîtrise de l'AH & 13 & 7 & 3 & & 3 \\
\hline Maîtrise moyenne de l'AH & 13 & 3 & 4 & 3 & 3 \\
\hline Bonne maîtrise de l'AH ${ }^{1 !}$ & 8 & & 1 & 7 & \\
\hline $0 \mathrm{~kg}$ concentré au pâturage & 8 & 6 & 1 & & 1 \\
\hline 1 à $2 \mathrm{~kg} / \mathrm{j}$ au pâturage & 12 & 2 & 2 & 4 & 3 \\
\hline 4 à $6 \mathrm{~kg} / \mathrm{j}$ au pâturage & 5 & & 2 & & 2 \\
\hline Distribution raisonnée au pâturage & 9 & $2(\mathrm{f})$ & 1 & 6 & \\
\hline Aire de couchage béton & 12 & & 6 & 2 & 4 \\
\hline Aire de couchage bois & 9 & 8 & 1 & & \\
\hline Aire de couchage béton paillé & 3 & & & 3 & \\
\hline Aire de couchage caoutchouc & 10 & 2 & 1 & 5 & 2 \\
\hline Traite manuelle & 5 & 5 & & & \\
\hline Lavage avec lessive & 9 & 4 & & 3 & 2 \\
\hline Lavage lessive + acide & 20 & 1 & 8 & 7 & 4 \\
\hline Pas nettoyage mamelle & 6 & 1 & 1 & 1 & 3 \\
\hline Essuie mamelle & 17 & 8 & 1 & 6 & 2 \\
\hline Lavage eau tiède + essuie & 7 & 1 & 3 & 2 & 1 \\
\hline Eau javelisée + essuie chiffons & 4 & & 3 & 1 & \\
\hline Nettoyage mamelle variable & 11 & 2 & 3 & 1 & 5 \\
\hline Même pratique à chaque traite & 23 & 8 & 5 & 9 & 1 \\
\hline Horaire de traite variable & 19 & 2 & 6 & 5 & 6 \\
\hline Horaire de traite régulier & 15 & 8 & 2 & 5 & \\
\hline
\end{tabular}

(f) : fabrication fermière

(1) la maîtrise de l'alimentation hivernale est définie à partir de la qualité de la ration de base (proportion de regain et apport d'aliment énergétique), du raisonnement de l'apport de concentré et du complément minéral vitaminisé.

Tableau 3. Caractéristiques des types de lait livrés $(n=129)$.

\begin{tabular}{|c|c|c|c|c|c|c|c|c|c|c|}
\hline $\begin{array}{l}\text { Type de lait } \\
\text { Critère }\end{array}$ & Classe & $\begin{array}{l}1 \\
7\end{array}$ & $\begin{array}{c}2 \\
27\end{array}$ & $\begin{array}{l}\text { variante } 2 \\
\quad 8\end{array}$ & $\begin{array}{c}3 \\
12\end{array}$ & $\begin{array}{l}\text { variante } 3 \\
6\end{array}$ & $\begin{array}{c}4 \\
15\end{array}$ & $\begin{array}{c}5 \\
17\end{array}$ & $\begin{array}{c}6 \\
15\end{array}$ & $\begin{array}{c}7 \\
22\end{array}$ \\
\hline $\begin{array}{l}\text { Structure } \\
\text { de livraison }\end{array}$ & $\begin{array}{l}\text { LV1 } \\
\text { LV2 } \\
\text { LV3 } \\
\text { LV4 } \\
\text { LV5 } \\
\text { LV6 } \\
\end{array}$ & $\begin{array}{l}6 \\
1\end{array}$ & & $\begin{array}{c}28 \\
5 \\
1 \\
1\end{array}$ & & $\begin{array}{c}4 \\
10 \\
4\end{array}$ & $\begin{array}{l}2 \\
6 \\
6 \\
\\
1 \\
\end{array}$ & $\begin{array}{l}4 \\
2 \\
8 \\
3\end{array}$ & $\begin{array}{c}3 \\
10 \\
1 \\
1 \\
\end{array}$ & $\begin{array}{c}1 \\
11 \\
9 \\
1\end{array}$ \\
\hline Volume annuel (kg) & $\begin{array}{l}\text { moyenne } \\
\text { écart-type }\end{array}$ & $\begin{array}{l}18692 \\
11733 \\
\end{array}$ & & $\begin{array}{l}12862 \\
9438 \\
\end{array}$ & & $\begin{array}{l}31250 \\
17034 \\
\end{array}$ & $\begin{array}{l}22035 \\
17496 \\
\end{array}$ & $\begin{array}{c}137397 \\
54928 \\
\end{array}$ & $\begin{array}{l}93824 \\
38834 \\
\end{array}$ & $\begin{array}{l}69718 \\
84126 \\
\end{array}$ \\
\hline $\begin{array}{l}\text { Taux protéique } \\
(\mathrm{g} / \mathrm{kg})\end{array}$ & $\begin{array}{l}\text { moyenne } \\
\text { écart-type } \\
\end{array}$ & $\begin{array}{c}28,6 \\
0,7 \\
\end{array}$ & & $\begin{array}{c}30,5 \\
1,8 \\
\end{array}$ & & $\begin{array}{c}30,7 \\
0,7 \\
\end{array}$ & $\begin{array}{c}29,8 \\
1,2 \\
\end{array}$ & $\begin{array}{c}31,6 \\
0,7 \\
\end{array}$ & $\begin{array}{c}31,3 \\
1 \\
\end{array}$ & $\begin{array}{c}30,8 \\
1,4 \\
\end{array}$ \\
\hline $\begin{array}{l}\text { Taux butyreux } \\
(\mathrm{g} / \mathrm{kg})\end{array}$ & $\begin{array}{l}\text { moyenne } \\
\text { écart-type }\end{array}$ & $\begin{array}{c}32,5 \\
1,2 \\
\end{array}$ & & $\begin{array}{c}37,1 \\
2,2 \\
\end{array}$ & & $\begin{array}{c}36,7 \\
1,9 \\
\end{array}$ & $\begin{array}{c}37,7 \\
1,8 \\
\end{array}$ & $\begin{array}{c}36,2 \\
1,2 \\
\end{array}$ & $\begin{array}{c}36,4 \\
1,8 \\
\end{array}$ & $\begin{array}{c}37,6 \\
1,9 \\
\end{array}$ \\
\hline $\begin{array}{l}\text { Propreté } \\
\text { leucocytaire }\end{array}$ & $\begin{array}{l}\text { LE1 } \\
\text { LE2 } \\
\text { LE3 } \\
\text { LE4 } \\
\text { LE5 } \\
\end{array}$ & 7 & & $\begin{array}{l}11 \\
11 \\
9 \\
4\end{array}$ & $\begin{array}{l}6 \\
6\end{array}$ & $\begin{array}{l}1 \\
5\end{array}$ & $\begin{array}{c}14 \\
1\end{array}$ & $\begin{array}{l}4 \\
6 \\
4 \\
\\
3\end{array}$ & $\begin{array}{l}4 \\
9 \\
2\end{array}$ & $\begin{array}{c}6 \\
11 \\
5 \\
\end{array}$ \\
\hline $\begin{array}{l}\text { Propreté } \\
\text { bactériologique }\end{array}$ & $\begin{array}{l}\text { BA1 } \\
\text { BA2 } \\
\text { BA3 } \\
\text { BA4 }\end{array}$ & $\begin{array}{l}2 \\
4 \\
1\end{array}$ & 27 & $\begin{array}{l}3 \\
5\end{array}$ & $\begin{array}{l}2 \\
7 \\
1 \\
2\end{array}$ & 6 & $\begin{array}{l}4 \\
3 \\
5 \\
3\end{array}$ & $\begin{array}{l}7 \\
5 \\
5\end{array}$ & 15 & $\begin{array}{l}8 \\
9 \\
5\end{array}$ \\
\hline
\end{tabular}


Figure 1. Evolution des caractéristiques des laits selon leurs types.

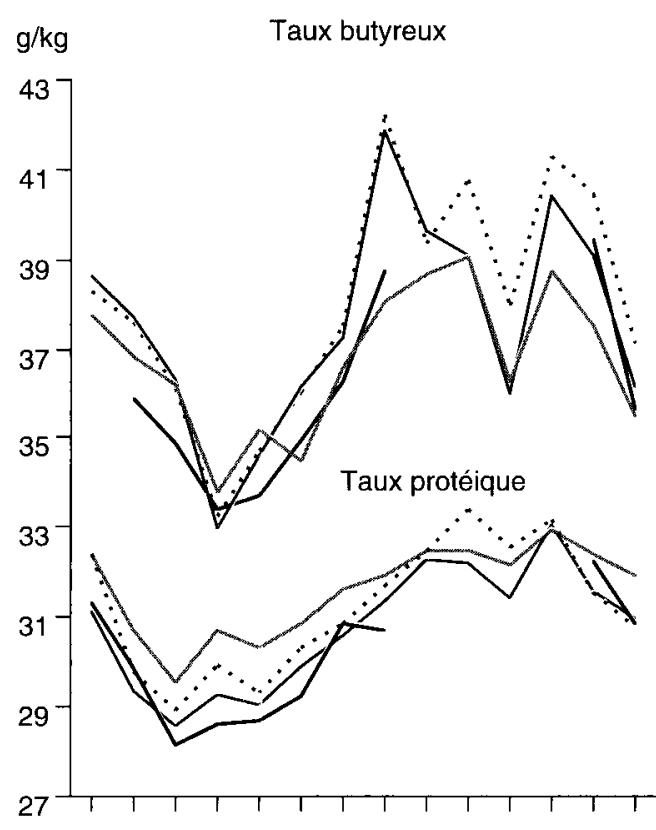

$N$ D J

\begin{abstract}
Lait livré $\left(10^{3} \mathrm{~kg} /\right.$ mois $)$
\end{abstract}
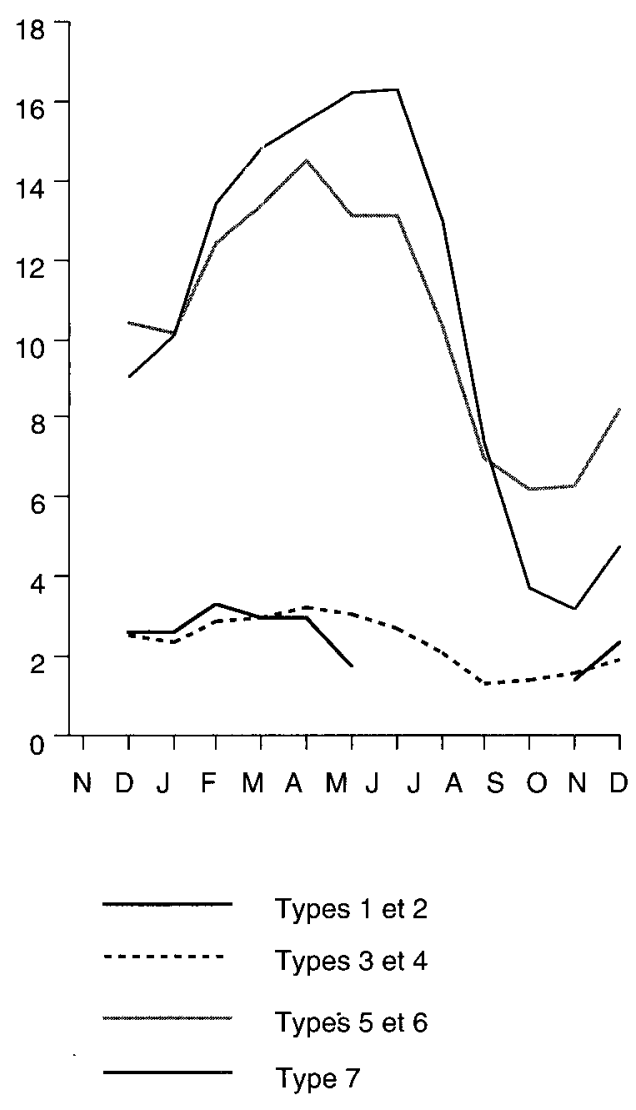

Germes totaux

(\% livraison)

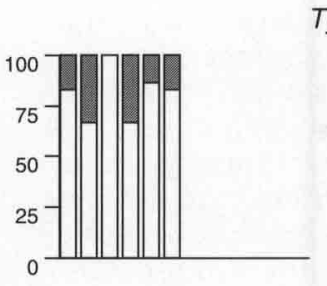

Type 2

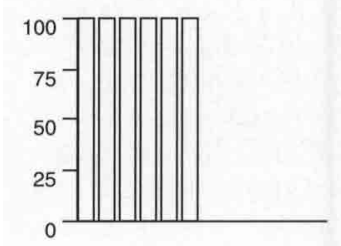

Type $3 b$

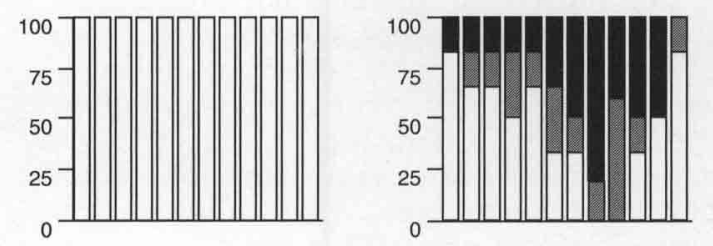

Type 4
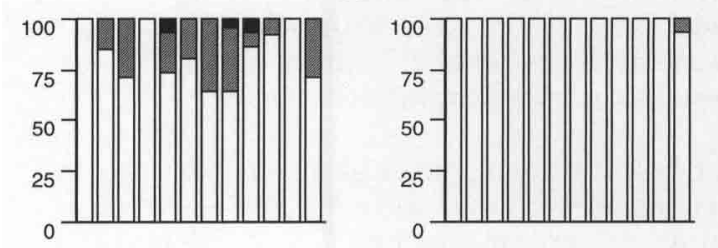

Type 5

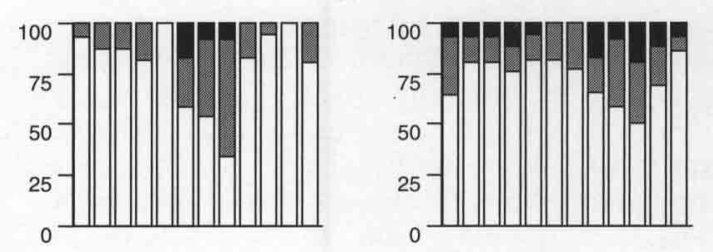

Type 6
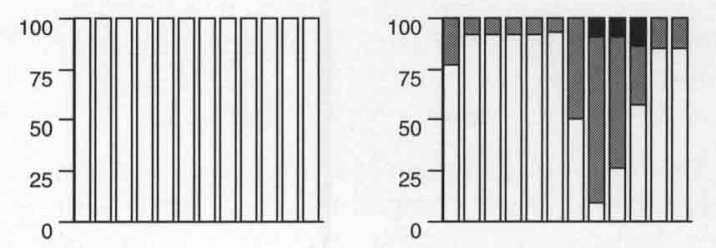

Type 7

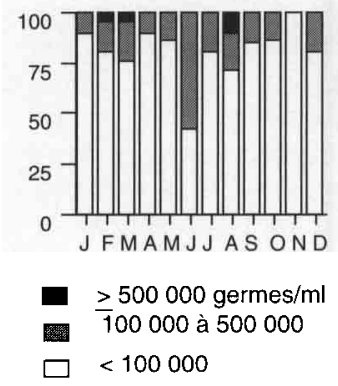

La contamination microbienne du lait est directement liée à l'hygiène de la traite, mais dépend aussi des conditions de logement des animaux. 
sante pour $77 \%$ des laits du type 2 . A l'opposé, elle est très élevée pour les autres laits (type 1 et variante 2 ).

L'arrêt de livraison de lait l'été est liée soit à la fabrication fermière du beaufort en alpage (2 exploitants sur 10 enquêtés), soit à la mise en pension du troupeau laitier de juin à la fin septembre ( 8 autres exploitants). Les évolutions des TP et TB moyens mensuels sont la conséquence des effets cumulés d'un regroupement fort des vêlages sur les mois de novembre à janvier et d'une alimentation hivernale mal maîtrisée (apport limité de fourrage de l'ordre de 10 à $12 \mathrm{~kg} / \mathrm{VL} / \mathrm{j}$ et quantité faible de concentré de 2 à $4 \mathrm{~kg} / \mathrm{VL} / \mathrm{j}$ ). Une proportion de regain plus importante et l'apport d'un aliment énergétique associé au concentré de production (VL18) limitent la baisse des taux du lait de type 2 durant la période hivernale. Le nettoyage succinct et irrégulier de la mamelle est un risque de contamination du lait et l'utilisation d'un plancher comme litière semble propice au développement de certains germes de l'environnement (7 exploitations livrant un lait de type 2). L'emploi d'un tapis en caoutchouc comme litière limite cette contamination (2 exploitations du type 1 ). La traite manuelle est associée à une faible teneur en germes totaux (4 exploitations). Le lavage de la machine à traire sans utilisation d'acide est associée en revanche à une forte teneur en germes totaux (3 exploitations sur 4).

Les laits des types 3 et 4 sont livrés toute l'année avec un maximum en mai et un minimum en septembre. Les TP et TB moyens mensuels sont les plus élevés en septembre (fin de lactation). Ils chutent de $5 \mathrm{~g} / \mathrm{kg}$ pour atteindre respectivement $28 \mathrm{~g} / \mathrm{kg}$ et $35 \mathrm{~g} / \mathrm{kg}$ en décembre-janvier (figure 1). Les taux remontent rapidement dès le mois suivant pour le type 3 , en avril-mai pour le type 4 . La teneur en germes totaux est élevée l'été dans les laits du type 4 et dans $67 \%$ des laits du type 3 , le tiers restant (variante 3 ) présentant une teneur satisfaisante toute l'année. Ces deux types se différencient par leur teneur en leucocytes (faible pour le type 4 , très élevée l'été pour le type 3 (figure 1).

Les 8 livreurs enquêtés maintiennent un effectif stable du troupeau laitier toute l'année. Les vêlages sont répartis principalement d'octobre à mars. La ration hivernale est composée de foin, de regain ou de « recosson» (foin après pâture) et d'un concentré distribué selon le niveau de production laitière. Les quantités de fourrages sont limités (10 à $12 \mathrm{~kg} / \mathrm{j}$ ), mais parfois compensées par un apport de betterave à partir de janvierfévrier, à une période où la plupart des vaches ont vêlé. Dans le lait de type 3 , l'augmentation du taux de leucocytes l'été est associée à des changements de pratiques de traite. D'une part les mamelles ne sont plus nettoyées l'été, alors qu'elles étaient lavées et essuyées l'hiver. D'autre part l'horaire de traite est variable à cette période. Les livreurs de lait du type 4 respectent une bonne hygiène de la mamelle (nettoyage avec de l'eau javellisée à l'aide de plusieurs chiffons voire de lavettes individuelles, séchage et trempage des trayons). La teneur satisfaisante du lait en germes totaux (variante 3 ) s'observe dans trois exploitations où est effectué un bon nettoyage de la machine à traire (utilisation d'acide 2 fois/semaine, brossage du matériel de traite).

Les laits des types 5 et 6 sont caractérisés par un volume annuel élevé, une bonne stabilité du TP moyen mensuel l'hiver et une teneur élevée en leucocytes durant les mois d'août à novembre. Si le lait de type 6 est livré plus particulièrement en hiver et au printemps et présente une bonne qualité bactériologique, celui du type 5 enregistre le volume livré maximal en juillet et sa teneur en germes totaux augmente en période estivale (juin à août) (figure 1).

Les 10 livreurs enquêtés regroupent les vêlages sur deux périodes, l'hiver et le printemps. Trois prennent des vaches en pension l'été pour augmenter l'effectif de leur troupeau durant la période d'alpage. Ces pratiques permettent une augmentation du volume de lait livré jusqu'en juillet. La stabilité du TP mensuel toute l'année est liée à la répartition des vêlages et aux pratiques d'alimentation hivernale : proportion importante de regain, apport d'un complément énergétique (betterave ou céréales) et distribution de concentrés selon le niveau de production. La recherche d'un fourrage de qualité incite trois éleveurs à acheter des regains en provenance de Crau. L'hygiène de la mamelle reste sommaire : simple essuyage ou lavage à l'eau et essuyage. L'utilisation de l'acide une à deux fois/semaine est systématique dans le cycle de lavage de la machine à traire chez les livreurs du type 6 , alors qu'elle ne concerne que deux producteurs du type 5 . Chez les livreurs du type 5 , les problèmes d'hygiène sont accrus l'été en raison de la difficulté rencontrée pour refroidir le lait en alpage ou d'intervalles de temps parfois importants entre la fin de traite et le ramassage du lait (30 à 45 minutes).

Le lait du type 7 est défini principalement par les critères sanitaires : le taux cellulaire et la concentration de germes totaux sont élevés toute l'année et particulièrement l'été (figure 1). Mais le TP moyen annuel, le volume annuel et le pourcentage de lait d'été présentent une variabilité intra-type importante.

Chez les six livreurs enquêtés, on observe plusieurs pratiques pouvant induire des problèmes d'hygiène (pas de nettoyage de la mamelle ou succinct pour cinq d'entre eux, pas d'emploi d'acide ou uniquement l'hiver dans le lavage de la machine à traire pour trois producteurs), mais aussi pouvant développer une sensibilisation de la mamelle à la contamination microbienne (trois pratiquent la mise en pension l'hiver des vaches taries, trois ont des vêlages en alpage et pour quatre les horaires de traite sont variables). 


\section{2 / Relations entre les types de lait et les systèmes d'exploitation}

L'AFC sur les pratiques d'élevage a fait apparaître des cohérences entre certaines pratiques. Les associations des pratiques d'agrégation, de répartition des vêlages et d'achats de fourrage ont constitué la trame d'identifica- tion des systèmes d'exploitation. Mais l'analyse des autres pratiques (alimentation, fertilisation, renouvellement...) et la connaissance de certains facteurs d'exploitation (présence d'alpage, importance du troupeau laitier, fabrication fermière...) ont été nécessaires pour comprendre leurs fonctionnements. Les 34 producteurs enquêtés ont été classés dans cinq systèmes d'exploitation (tableau 4).

Tableau 4. Les systèmes d'exploitation.

\begin{tabular}{|c|c|c|c|c|c|}
\hline $\begin{array}{l}\text { Système d'exploitation } \\
\text { Effectif }(n=34)\end{array}$ & $\begin{array}{c}\text { S1a } \\
8\end{array}$ & $\begin{array}{c}\text { S1b } \\
3\end{array}$ & $\begin{array}{l}\text { S2 } \\
11\end{array}$ & $\begin{array}{c}\mathbf{S 3} \\
\mathbf{9}\end{array}$ & $\begin{array}{c}\text { S4 } \\
3\end{array}$ \\
\hline Caractéristiques des exploitations (min-max) & & & & & \\
\hline Effectif vaches laitières & 3 à 17 & 25 à 42 & 8 à 38 & 30 à 83 & 40 à 110 \\
\hline Quota laitier (en milliers l) & 20 à 30 & 110 à 140 & 21 à 110 & 100 à 250 & 80 à 300 \\
\hline Surface du pôle du bas (ha) & 10 à 20 & 40 & 11 à 32 & 10 à 50 & 7 à 23 \\
\hline Surfaces des montagnettes (ha) "' & 3 à 13 & 0 à 10 & 0 à 4 & 25 à 50 & 10 à 20 \\
\hline Alpage & non & non & variable & oui & oui \\
\hline Mise à l'estive du troupeau & 8 & 3 & & & \\
\hline Stabilité du troupeau laitier & & & 11 & & 1 \\
\hline Prise à l'estive des laitières & & & & 5 & \\
\hline Mise à l'hiverne des laitières & & & & 4 & 2 \\
\hline Pas d'achat de foin & 6 & 1 & 7 & & \\
\hline Achète du foin de pays & 2 & 2 & 4 & 1 & \\
\hline Achète du foin de Crau & & & & 8 & 3 \\
\hline Autonomie fourragère & 8 & 3 & 10 & & \\
\hline $50 \%$ à $80 \%$ d'autonomie & & & 1 & 8 & \\
\hline 0 à $40 \%$ d'autonomie & & & & 1 & 3 \\
\hline Vêlage d'octobre février & 8 & & 5 & & \\
\hline Vêlage d'octobre à mars & & 3 & 5 & & \\
\hline Vêlage d'octobre à juin & & & 1 & 9 & \\
\hline Vêlage toute l'année & & & & & 3 \\
\hline Pas d'alpage & 8 & 3 & 4 & & \\
\hline Pratique de l'inalpage & & & 7 & 9 & 3 \\
\hline Fumier sur pré de fauche & 6 & & 4 & 4 & 3 \\
\hline Engrais sur pré de fauche & 2 & & 3 & 4 & \\
\hline Engrais sur pré de fauche et pâturage & & 3 & 4 & 1 & \\
\hline Mauvaise maitrise de l'alimentation hivernale & 5 & & 4 & 3 & 1 \\
\hline Maîtrise moyenne de l'alimentation hivernale & 3 & & 6 & 3 & 2 \\
\hline Bonne maîtrise de l'alimentation hivernale & & 3 & 1 & 3 & \\
\hline Pas de complémentation au pâturage & 5 & & 4 & & 1 \\
\hline 1 à $2 \mathrm{~kg} / \mathrm{j}$ & 3 & 1 & 4 & 5 & \\
\hline 4 à $6 \mathrm{~kg} / \mathrm{j}$ & & & 1 & & 2 \\
\hline Distribution raisonnée & & 2 & 2 & 4 & \\
\hline Traite manuelle & 5 & & & & \\
\hline Lavage avec lessive & 3 & & & 5 & 2 \\
\hline Lavage lessive + acide & & 3 & 11 & 4 & 1 \\
\hline Pas de nettoyage de la mamelle & 1 & & 1 & 2 & 2 \\
\hline Essuie (mamelle) & 5 & 3 & 2 & 6 & 1 \\
\hline Lavage eau tiède + essuie & 2 & & 5 & & \\
\hline Eau javellisée + essuie chiffons & & & 3 & 1 & \\
\hline Même pratique à chaque traite & 6 & 3 & 8 & 6 & \\
\hline Nettoyage mamelle variable & 2 & & 3 & 3 & 3 \\
\hline Horaire de traite régulier & 7 & 1 & 6 & 5 & \\
\hline Horaire de traite variable & 1 & 2 & 5 & 4 & 3 \\
\hline Fabrication fermière l'été & & & & 2 & \\
\hline
\end{tabular}

(1) La montagnette est un ensemble de parcelles situées à une altitude intermédiaire entre le siège d'exploitation et l'alpage, qui sont destinées au pâturage au printemps et à l'automne. 
Onze pratiquent la mise en pension l'été du troupeau laitier, produisent leur fourrage durant la période estivale et regroupent les vêlages à l'automne et en début d'hiver pour valoriser au maximum le lait durant la période où les vaches sont sur l'exploitation. On distingue toutefois deux systèmes d'exploitation : le traditionnel (S1a) et l'intensif (S1b). Ces deux systèmes se différencient par l'importance des moyens de production (quotas, surfaces exploitées, effectif du troupeau...) et des intrants utilisés (engrais, concentrés...).

Onze autres exploitants gardent leur troupeau laitier toute l'année sur l'exploitation, permettant ainsi d'avoir des vêlages plus précoces à l'automne dès le mois d'octobre et de les étaler jusqu'en mars. Ils restent toutefois dans une logique de production de lait d'hiver pour produire leur foin durant l'été (système d'exploitation S2).

Neuf exploitants sont dans une logique de production laitière orientée l'été. Ils répartissent les vêlages d'octobre à juin et augmentent l'effectif de leur troupeau laitier en période estivale en prenant des vaches en pension et/ou en réintroduisant au printemps leurs vaches mises en pension durant l'hiver. Comme il est difficile de concilier l'autonomie fourragère et la production laitière en alpage, ces exploitants achètent 20 à $50 \%$ de leur fourrage (système d'exploitation S3). Deux exploitants fabriquent du Beaufort l'été et ne livrent donc le lait à la coopérative que durant la période allant d'octobre à mi-juin.

Enfin trois gros alpagistes achètent de 60 à $80 \%$ de leur fourrage pour l'alimentation hivernale du troupeau (60 vaches environ). Les exploitants mettent toutefois de 20 à $30 \%$ des vaches en pension l'hiver, de préférence les taries qui vêleront au printemps. Les vêlages ont lieu toute l'année sauf en août et en septembre (système d'exploitation S4).

La mise en relation des systèmes d'exploitation et des types de laits font apparaître des liaisons fortes entre eux (figure 2). En effet, des types de laits sont associés à des pratiques dont les liens sont imputables au système d'exploitation et qui conduisent à des associations de caractéristiques de lait. Par exemple, les types de lait 1 et 2 résultent principalement de deux pratiques liées, la mise à l'estive des laitières et le fort regroupement des vêlages à l'automne, et qui sont associées à des pratiques d'alimentation hivernale mal maîtrisée. L'ensemble de ces pratiques sont mises en œuvre dans le système d'exploitation S1a. De même les pratiques associées aux types de lait 3 et 4 concourent à la logique du système d'exploitation $\mathrm{S} 2$.

Les relations entre pratiques et caractéristiques de lait apparaissent, de plus, complexes en raison des associations multiples existant entre elles. La prise à l'estive de vaches laitières, par exemple, contribue à expliquer en partie les caractéristiques suivantes: livraison permanente avec un fort pourcentage de lait d'été, volume important
Figure 2. Relations entre les types de lait et les systèmes d'exploitation.

Nombre d'exploitations

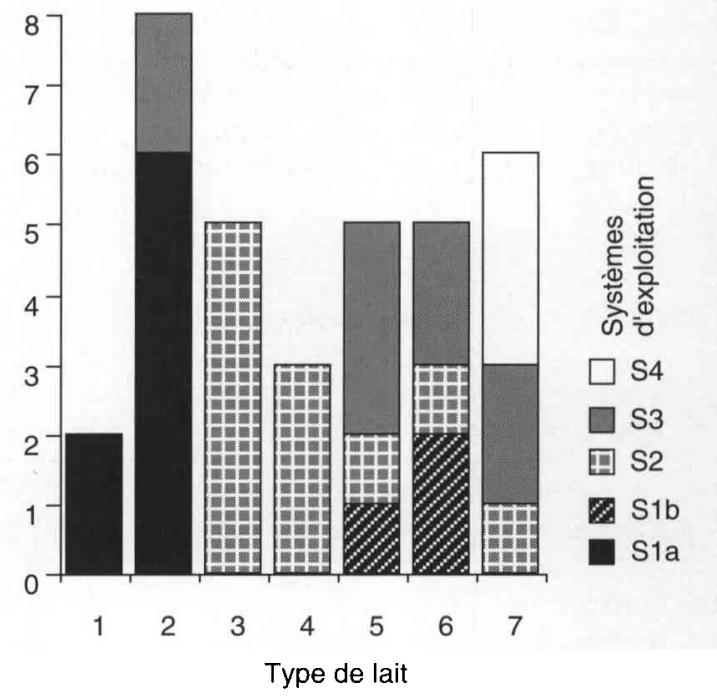

de lait livré, TB annuel élevé et taux de leucocytes élevé l'été. L'ensemble de ces caractéristiques contribuent à définir le type de lait 5 .

$1 l$ peut également exister une diversité de pratiques permettant de satisfaire une même logique de production. Le système d'exploitation S3 développe une logique de production laitière orientée l'été. L'augmentation du pourcentage de lait d'été peut être attribuée à des pratiques diverses et parfois associées : pratiques d'agrégation et/ou de répartition de vêlages et/ou d'alimentation. Or ces pratiques entraînent des conséquences variées sur d'autres caractéristiques de lait (composition chimique, qualité sanitaire), qui entrent dans la définition du type de lait. Par exemple, les pratiques de mise en pension l'hiver et/ou de prise en pension l'été, associées au pourcentage de lait d'été, sont repérées comme facteur de risque de mammites. Aussi trois exploitations qui ont la même logique de production laitière (système d'exploitation S3) peuvent livrer un type de lait différent $(5,6$ ou 7$)$.

Inversement, un type de lait est parfois associé à plusieurs logiques de fonctionnement d'exploitation. Ceci s'explique par le fait que les critères caractérisant le type de lait sont liés à la capacité du producteur à maîtriser les facteurs relatifs à la conduite du troupeau : facteurs alimentaires maîtrisés (types de lait 5 et 6 ), facteurs d'hygiène non maîtrisés (type 7). Ce résultat confirme que les contraintes du système d'exploitation (absence d'alpage, par exemple) ne sont pas les premiers facteurs limitants de certaines caractéristiques du lait, telle que sa composition chimique, qui incombent bien plus à la capacité du producteur à maîtriser des facteurs du système de production. Ceci a déjà été montré pour la production laitière (Dobremez et al 1989 et 1990, Coulon et al 1990). 


\section{3 / Discussion}

Cette étude confirme, pour la composition chimique du lait, les connaissances expérimentales et les observations faites dans d'autres situations: la période de vêlage (Coulon et al 1988, Agabriel et al 1990) et le niveau des apports énergétiques de la ration (Hoden et al 1985. Sutton 1989, Coulon et Rémond 1991) expliquent les évolutions de la composition chimique du lait très différentes entre les types de lait. Les laits à faible TP annuel (1 et 4 ) s'expliquent par les pratiques alimentaires hivernales conduisant à des apports énergétiques insuffisants, surtout en période de début de lactation. Chez les exploitants livrant du lait à fort TP annuel et où l'évolution du TP au cours de l'année est la plus régulière ( 5 et 6 ), les facteurs alimentaires semblent maîtrisés. L'importance des variations du TB sous l'effet des facteurs alimentaires et saisonniers (Agabriel et al 1990, Agabriel et al 1993) amène une variabilité intra-type du TB annuel plus importante que pour le TP. Les valeurs des TP et TB annuels s'avèrent, de plus, peu liés. Les laits à faible TP annuel ont des TB annuels très variables : élevé pour le type 4 , faible pour le type 1 . Ces laits sont produits dans des exploitations où les vêlages sont regroupés à l'automne et en début d'hiver ; contrairement aux exploitations livrant du lait de type 1 , celles livrant le lait de type 4 maintiennent un troupeau de vaches laitières de fin de lactation, d'où une augmentation importante du TB (Rémond 1987, Schultz et al 1990). Les laits à fort TB annuel (types 4 et 7 ) présentent des augmentations importantes du TB mensuel en juin et juillet. Ceci s'explique en partie par l'importance des vêlages (10 à $20 \%$ des vaches) à cette période chez les exploitants livrant le lait du type 7. Toutefois les effets conjugués de la qualité de l'herbe et du stade de lactation peuvent expliquer cette augmentation importante du taux butyreux (Coulon et al, 1991).

L'étude fait également apparaître une variabilité inter-type de la qualité hygiénique du lait (cellules et germes totaux). Les laits de type 2 , variante 3 et 6 présentent des taux cellulaires élevés et peu de germes totaux. Bakken (1981) a également constaté des taux cellulaires élevés dans le lait en l'absence apparente de contaminations bactériennes. Tous ces types de laits sont associés à des pratiques de nettoyage succinct et irrégulier de la mamelle favorisant la contamination des trayons lors de la traite (Galton et al 1986, 1988). L'augmentation importante de leucocytes en été, mise en évidence dans d'autres études (Simensen 1974, Dohoo et al 1984, Goldberg et al 1992), est liée en partie au stress provoqué par les variations d'horaires de traite durant la période de fenaison. La mise en pension l'hiver des vaches taries, réintroduites dans le troupeau au moment du vêlage, est également un facteur de risque de mammites, en raison de l'effet propre du vêlage (Brochart et al 1984, Barnouin et al
1986 ) et du changement brutal d'environnement microbien (Faye et al 1994). La faible teneur en germes totaux dans ces laits s'explique principalement par l'hygiène du matériel de traite : la pratique de la traite manuelle ou l'emploi périodique de l'acide détruisant la flore thermorésistante (Alais 1984) diminue la contamination du lait par la machine à traire (Fell et al 1964, McDonald 1969, Galton et al 1982, O'Mahony et al 1991). Sur les 10 producteurs enquêtés n'utilisant pas d'acide dans le cycle de lavage de leur machine à traire, 8 livrent un lait chargé en germes totaux. Certains exploitants livrant du lait l'été sont de plus confrontés au problème du refroidissement du lait durant l'inalpage. La présence, en quantité parfois importante, de germes totaux dans les laits ne suffit pas à expliquer les infections mammaires responsables des taux élevés de leucocytes (Bramley et al 1984). Ces types de laits sont associés à des facteurs de risques de mammites : nettoyage succinct de la mamelle pour $71 \%$ d'entre eux, variabilité des pratiques de traite (horaire, nettoyage de la mamelle et du matériel de traite...) pour les livreurs du type 7 , changements brutaux d'environnement microbien (mise à l'hiverne pour le type 7, prise à l'estive pour le type 5), vêlages en été (type 7) conduisant à des augmentations de mammites post-partum (Faye et al 1986, Collier et al 1992). Certaines pratiques limitent la contamination microbienne des trayons. Les trois livreurs du type 4 (caractérisé par une teneur faible en leucocytes et par la présence de germes) utilisent de l'eau javellisée pour nettoyer les mamelles et emploient plusieurs chiffons voire parfois de lavettes individuelles permettant de garantir une meilleure propreté des trayons et de réduire ainsi la population bactérienne (Sheldrake et al 1980, Barnouin et al 1986, Pankey 1989). Dans les deux exploitations livrant le lait de type 1 (présentant les mêmes caractéristiques que le type 4), l'aire de couchage en caoutchouc des laitières préserve les trayons d'éventuels traumatismes (Brochart et al 1982, cité par Faye 1986) et semble moins favorable au développement de germes de l'environnement que les litières à base de sciure de bois (Keller et Jonggaard 1985 cité par Faye 1986, Schukken et al 1991) ou les litières paillées (Rendos et al 1975).

Ces résultats montrent une hétérogénéité importante et une maîtrise souvent partielle des pratiques d'hygiène dans les exploitations. Le respect de l'hygiène du matériel de traite n'est pas associé systématiquement à une bonne propreté de la mamelle.

L'hétérogénéité dans la maitrise de l'hygiène s'observe également au niveau du système d'élevage. La typologie des laits livrés montre des associations variées de leurs caractéristiques, parfois peu courantes, sous-tendant une diversité des pratiques associées et de la maîtrise des producteurs. Les TP et TB des laits s'avèrent peu liés à la teneur en germes totaux et en leucocytes : il existe, en effet, une grande diversité d'associations de ces cri-
La mâ̂trise des pratiques d'hygiène est souvent partielle dans les exploitations. 
tères selon les types de laits. La comparaison des pratiques associées aux différents types de laits montre que les producteurs maîtrisent rarement l'ensemble des facteurs du système d'élevage. L'application des règles prophylactiques concernant les mammites cliniques (lavage de la mamelle avec des lavettes individuelles, entretien de la machine à traire, litière...) n'est pas associée à des facteurs alimentaires maîtrisés dans les exploitations livrant le lait de type 4 . Inversement, les producteurs livrant le lait de type 6 maîtrisent mieux l'alimentation hivernale que les pratiques permettant de minimiser les risques d'infections mammaires. Dans les exploitations où les facteurs alimentaires semblent maîtrisés, il existe également une grande variabilité dans l'efficacité des pratiques d'hygiène, conduisant à des différences de teneur en germes totaux entre les types 5 et 6 . Ces résultats tendent à montrer un déséquilibre entre la capacité des producteurs à maîtriser l'évolution technique de leur exploitation et leur capacité à mettre en œuvre des pratiques sanitaires.

La mise en relation des types de lait avec les systèmes d'exploitation confirme l'importance des liaisons entre des "profils de performances " et des systèmes d'exploitation (Faye et al 1991, Moulin 1993), mais en montre également la complexité. La diversité des laits livrés traduit celle des objectifs de production et des pratiques ainsi que l'hétérogénéité existant au niveau de la maîtrise des producteurs. Se pose donc la question de la prise en compte de cette complexité dans le conseil en matière de qualité du lait. Le raisonnement qui consiste à apporter une solution normative à un problème de qualité du lait (résolution du problème grâce à la connaissance de ses facteurs de variation) fait abstraction de la diversité des systèmes d'exploitation et des pratiques mises en œuvre par les producteurs pour satisfaire leur logique de production laitière. Ceci contribue au développement d'un modèle de production. Le raisonnement des problèmes de qualité dans la logique globale de l'exploitation permet, en revanche, de proposer des actions de qualité adaptées à la diversité des exploitations laitières et donc la préserve. Connaître les pratiques imputables au système d'exploitation parmi celles qui sont associées aux types de lait permet d'anticiper les effets des actions de qualité sur les systèmes d'exploitation.

La connaissance du système d'exploitation à travers l'ensemble des pratiques permet également d'associer aux caractéristiques des laits livrés des critères reflétant le caractère "d'authenticité " du Beaufort (lien au terroir, cohérence des fonctionnements, occupation de l'espace rural...). Par exemple, l'occupation de l'espace s'avère différente entre systèmes d'exploitation, dans la mesure où la logique de production influe sur le nombre de pôles fonciers exploités, l'altitude des espaces utilisés et plus globalement le fonctionnement fourrager (Dubeuf et al 1995, Fleury et al 1995).

\section{Conclusion}

Cette étude s'inscrit dans une problématique de recherche-action qui renvoie non seulement à la connaissance de performances et de leur élaboration mais aussi à l'organisation de filière et des conventions de qualité (Eymard-Duvernay 1989) entre les coopératives et leurs producteurs. En effet, l'identification des attentes des coopératives en matière de qualité du lait et l'analyse des conséquences de leurs règles d'actions de qualité sur les relations avec leurs producteurs sont des éléments fondamentaux à intégrer dans une démarche de conseil de qualité.

Les résultats de cette étude, enrichis par ceux sur les laits livrés dans trois autres coopératives, sont actuellement utilisés pour élaborer un mode de raisonnement d'une démarche de qualité adaptée à la diversité des situations à l'échelle d'une coopérative. Celle-ci identifie un groupe de livreurs par le type de lait livré et son système d'exploitation. Chaque groupe de livreurs est repéré par rapport à des problèmes de qualité du lait que la coopérative exprime. Un problème de qualité est analysé en termes d'objectifs prioritaires à résoudre, de faisabilité et de niveau d'intervention, qui se pose en termes d'intervenants (coopérative, UPB, organisme de développement...) et d'interventions (conseil personnalisé, action collective, incitations...).

\section{Remerciements}

L'auteur remercie le Directeur de l'Union des Producteurs de Beaufort et les Présidents et Directeurs des coopératives fromagères qui ont permis la réalisation de ce travail, ainsi qu'Agnès Hauwuy pour sa collaboration. Merci enfin à Jean-Baptiste Coulon et Etienne Landais pour leurs conseils constructifs.

Ce travail a été réalisé dans le cadre du programme de Recherche-Développement des Alpes du Nord (SUACI, 11 rue métropole, 73000 Chambéry)

\section{Références bibliographiques}

Agabriel C., Coulon J.B., Marty G., Cheneau N., 1990. Facteurs de variation du taux protéique du lait de vache. Etude dans les exploitations du Puy de Dôme. INRA Prod. Anim., 3, 137-150.
Agabriel C., Coulon J.B., Marty G., Bonaiti B., 1993. Facteurs de variation de la composition chimique du lait dans les exploitations à haut niveau de production. INRA Prod. Anim., 6, 53-60. 
Alais C., 1984. Science du lait. Principes des techniques laitières. Ed. SEPAIC ( $4^{\circ}$ édition) Paris. 814 pages.

Bakken G., 1981. Environment and bovine udder diseases in the loose housing system for dairy cows with reference to relevant data from the cowhouse system. Acta Agri Scand., 31, 445-451.

Barnouin J., Fayet J.C., Jay M., Brochart M., 1986 Enquête éco-pathologique continue : facteurs de risque des mammites de la vache laitière. I. Analyses multidimensionnelles sur données d'élevage. Can. Vet. J., 27, 135-145.

Bramley A.J. McKinnon C.H., Stker R.T., Simpkin D.L., 1984. The effect of udder infection on the bacteria flora of the bulk milk of ten dairy herds. J. Appl. Bact., 57, 317-323.

Brochart M., Barnouin J., Chassagne M., Faye B., Fayet J.C., 1984. Vaches laitières : contribution des enquêtes écopathologiques à l'établissement des programmes sanitaires d'élevage. In « Les entretiens de Bourgelat », Tome IV : 43-75. ENVL, Lyon.

Collier R.J., Beede D.K., Thatcher W.W., Israel L.A., Wilcox C.J., 1982. Influences of environment and its modification on dairy animal health and production. J. Dairy Sci., 65, 2213-2227.

Coulon J.B., Rémond B., 1991. Variations in milk output and milk protein content in response to level of energy supply to the dairy cow : a review. Livest. Prod. Sci., 29, 31-47.

Coulon J.B, Roybin D., Congy E., Garret A., 1988. Composition chimique et temps de coagulation du lait de vache : facteurs de variations dans les exploitations du pays de Thônes (Haute-Savoie). INRA Prod. Anim., 4, 49-56.

Coulon J.B., Roybin D., Cristofini B., 1990. Production laitière et fonctionnement d'exploitations : facteurs de variations dans les exploitations du Pays de Thônes (Haute-Savoie). INRA Prod. Anim., 3, 287 298.

Coulon J.B., Chilliard Y., Rémond B., 1991. Effet du stade physiologique et de la saison sur la composition chimique du lait de vache et ses caractéristiques technologiques. INRA Prod. Anim., 4, 219228.

Dobremez L., Baud G., Barret M., Roussel M., Liénard G., Lherm M., Pizaine M.C., 1989. Etude économique de la production laitière en moyenne montagne dans les exploitations de la Haute-loire. Campagne 1986-1987. Etude concertée 126, CEMAGREF (63200 Riom), INRA Economie de l'élevage (63122 Theix) et CERHL (43000 Le Puy).

Dobremez L., Baud G., Barret M., Roussel M., Liénard G., Lherm M., Pizaine M.C., 1990. Etude économique de la production laitière en moyenne montagne dans les exploitations de la Haute-loire. Campagne 1988-1989. Etude concertée 135, CEMAGREF (63200 Riom), INRA Economie de l'élevage (63122 Theix) et CERHL (43000 Le Puy).

Dohoo I., Martin S.W., MC Millan I., Kennedy B.W., 1984. Disease production and culling in HolsteinFriesian cows. II. Age, season and sire effects. Prev. Vet. Med., 2, 655-670.

Dubeuf B., Fleury P., Jeannin B., 1995. Diversité des fonctionnements fourragers et conséquences pour le conseil. Fourrages, 141.
Faye B., 1986. Facteurs de l'environnement et pathologie non-parasitaire de la vache laitière. Données bibliographiques et synthèse des résultats de l'enquête éco-pathologique continue. Bull. Tech. CRZV Theix, INRA, 64, 9-20.

Faye B., Brochart M., 1986. Enquête éco-pathologique continue : 7. Approche épidémiologique des mammites post-partum chez la vache laitière : étude descriptive et typologie des élevages. Ann. Rech. Vét., 1986, 17, 297-311.

Faye B., Grelet Y., 1991. Profils sanitaires en élevage bovin laitier. Mise en relation avec une typologie d'exploitation. Etudes et recherches sur les systèmes agraires et le développement, 21, 13-47.

Faye B., Dorr N., Lescourret F., Barnouin J., Chassagne M., 1994. Farming practices associated with the "udder infection " complex. Vet. Res., 25, 213-218.

Fell L.R., 1964. Machine milking and mastitis - a review. Dairy Sci. Abstr., 26, 551-557.

Fleury P., Dubeuf B., Jeannin B., 1995. Un concept pour le conseil en exploitation laitière : le fonctionnement fourrager. Fourrages, 141.

Galton D.M., Petersson L.G., Merrill W.G., 1986. Effects of premilking udder preparation practices on bacterial counts in milk and on teats. J. Dairy Sci., $69,260-266$.

Galton D.M., Petersson L.G., Merrill W.G., 1988. Evaluation of udder preparations on intramammary infections. J. Dairy Sci., 71, 1417-1422.

Goldberg J.J., Wildman E.E., Pankey J.W., Kunkel J.R., Howard D.B., Murphy B.M., 1992. The influence of intensively managed rotational grazing, traditional continuous grazing, and confinement housing on bulk tank milk quality and udder health. J. Dairy Sci., 75, 96-104.

Hoden A., Coulon J.B., Dulphy J.P., 1985. Influence de l'alimentation sur la qualité du lait. 3 . Effets des régimes alimentaires sur les taux butyreux et protéique. Bull. Tech. CRZV Theix, INRA, 2, 69-79.

Landais E., 1987. Recherches sur les systèmes d'élevage. Questions et perspectives. Document de travail de l'URSAD Versailles-Dijon-Mirecourt, INRASAD Versailles, $73 \mathrm{p}$.

Landais E., Deffontaines J.P., 1988. Les pratiques des agriculteurs. Point de vue sur un courant nouveau de la recherche agronomique. Economie rurale, 109, 125-158.

McDonald J.S., 1969. Relationship of hygiene, milking machine function and intramammary therapy to udder disease. J. Am. Vet. Med. Assoc., 170, 11961200.

Moulin C.H., 1993. Performances animales et pratiques d'élevage en Afrique sahélienne. La diversité du fonctionnement des troupeaux de petits ruminants dans la communauté rurale de Ndiagne (Sénégal). Thèse Doct. Ing. INA-PG. ENSSAA Dijon. $234 \mathrm{p}$.

Pankey J.W., 1989. Premilking udder hygiene. J. Dairy Sci., 72, 1308-1312.

Pluvinage P., Ducruet T., Josse J., Monicat F., 1991. Facteurs de risque des mammites des vaches laitières. Résultats d'enquête. Rec. Méd. Vét., 167, 105112. 
Rémond B., 1987. Influence du stade de lactation et de l'âge sur la composition chimique du lait. In "Le lait, matière première de l'industrie laitière ". INRA publications, Versailles, 151-159.

Rendos J.J., Eberhart R.J., Kesler E.M., 1975. Microbial populations of teat ends of dairy cows and bedding materials. J. Dairy Sci., 58, 1492-1500.

Schultz M.M., Hansen L.B., Steuernagel G.R., Kuck L., 1990. Variation of milk, fat, protein and somatic cells for dairy cattle. J. Dairy Sci., 72, 484-493.

Sheldrake R.F., Hoare R.J.T., 1980. Effect of a disinfectant udder wash and a post-milking teat dip on the bacterial population of the teat end and on the rate of new intramammary infection. J. Dairy Res., $47,253-258$.

Simensen E., 1974. The relationships between weather and incidence of parturient paresis and mastitis in dairy cows. Nord. Vet. Med., 26, 382-386.

Schukken Y. H., Grommers F.J., Van de Geer D., Erb H.N., Brand A., 1991. Risk factors for clinical mastitis in herds with a low bulk milk somatic cell count. 2. Risk factors for Escherichia coli and Staphylococcus aureus. J. Dairy Sci., 74, 826-832.

Sutton J.D., 1989. Altering milk composition by feeding. J. Dairy Sci., 2, 2801-2814.

\begin{abstract}
Relationships between milk characteristics, breeding practices and farming systems in area of Beaufort cheese's production.

A typology of 129 milk samples collected by two cooperatives to make Beaufort cheese, showed various associations in their milk characteristics (fat content, protein content, somatic cell count, bacteria count, breakdown of monthly milk bulk, annual milk bulk). Production practices were studied in 34 dairy farms. Feeding factors and calving periods were found to have an influence on the milk's chemical composition. The bacteria counts in milk depended on the sanitation of the milking equipment. Farming practices, premil-
\end{abstract}

Abstract king udder preparation and cubicle mats were associated with somatic cell count. The variability of the different milk characteristics and the relations amongst them underlines the diversity in both farming practices and the control systems currently in place. Relationships between milk types and farming systems demonstrates the utility of proposing quality guidelines that are adapted to the diversity of dairy farming.

DUBEUF B., 1995. Relations entre les caractéristiques des laits de troupeaux, les pratiques d'élevage et les systèmes d'exploitation dans la zone de production du Beaufort. INRA Prod. Anim., 8 (2), 105-116. 